\title{
HEMATOMAS E PETÉQUIAS INTESTINAIS ASSOCIADA A ENTERITE PROXIMAL EM EQUINO PORTADOR DE SÍNDROME CÓLICA
}

\author{
AGOSTINHO, Juliana Maria Avanci ${ }^{1}$ \\ FREITAS, Fernanda Coutinho de ${ }^{2}$ \\ LATARO, Rita Aparecida ${ }^{3}$ \\ MORAES, Angélica Trazzi Bento de ${ }^{4}$ \\ VALENTE, Paula Pimentel ${ }^{4}$
}

Recebido em: 2010.09.15 Aprovado em: 2010.10.28

ISSUE DOI: $10.3738 / 1982.2278-474$

\begin{abstract}
RESUMO:Caracterizada por manifestação de dor abdominal aguda, sendo mais comuns as dores de origem gastrointestinal, a Síndrome Cólica é uma das principais enfermidades que acometem a espécie equina. Como uma das causas de manifestações de dor abdominal aparece a enterite aguda, uma doença inflamatória de etiologia desconhecida, envolvendo principalmente o duodeno. O presente trabalho relata o caso de uma égua da raça quarto de milha atendida no Hospital Veterinário da FAFRAM apresentando sinais de desconforto abdominal com possível deslocamento do ceco. À laparotomia exploratória confirmou-se o deslocamento do ceco associado à presença de hematomas e petéquias em toda a extensão da serosa do intestino delgado, sugestivos de enterite proximal. O pós-operatório foi mantido com antibioticoterapia e uso de antiinflamatórios, medicações pró-cinéticas e protetores de mucosa gástrica. A complicação pós-operatória observada foi endotoxemia seguida de começo de laminite aguda, também controladas. Após o término da terapia medicamentosa os sinais de desconforto abdominal, endotoxemia e laminite cessaram, sendo a terapia clínica e cirúrgica satisfatórias.
\end{abstract}

Palavas-chave: Síndrome Cólica. Enterite Proximal. Equino.

\section{INTESTINAL HEMATOMAS AND PETECHIAE ASSOCIATED PROXIMAL ENTERITIS IN EQUINE WITH COLIC SYNDROME}

\begin{abstract}
SUMMARY:Characterized by manifestation of acute abdominal pain, the pain was more common source of gastrointestinal origin, the Colic Syndrome is one of the main diseases affecting the equine species. As a cause of abdominal pain manifestations appears to acute enteritis, an inflammatory disease of unknown etiology, involving mainly the duodenum. This paper reports the case of a horse race quarter mile seen at the Veterinary Hospital of FAFRAM showing signs of abdominal discomfort with the possible displacement of the cecum. At exploratory laparotomy confirmed the displacement of the cecum associated with the presence of bruising and petechiae over the entire length of the serosa of the small intestine, suggestive of proximal enteritis. The postoperative period was maintained with antibiotics and anti-inflammatory drugs, prokinetic drugs and protective of the gastric mucosa. e postoperative complication rate was followed by endotoxemia early acute laminitis, also controlled. After the drug therapy the signs of abdominal discomfort, endotoxemia and laminitis ceased, and the satisfactory clinical and surgical therapy.
\end{abstract}

Keywords: Syndrome Colic. Proximal Enteritis. Equine.

\footnotetext{
${ }^{1}$ Aprimoranda do Hospital Veterinário da FAFRAM

${ }^{2}$ Médica Veterinária do Setor de Clínica e Cirurgia de Grandes Animais do Hospital Veterinário da FAFRAM

${ }^{3}$ Médica Veterinária Anestesista do Hospital Veterinário da FAFRAM,

${ }^{4}$ Docente do Curso de Medicina Veterinária FAFRAM
} 


\section{INTRODUÇÃO}

A Síndrome Cólica é uma das principais enfermidades que acometem a espécie equina, sendo esta caracterizada por manifestação de dor abdominal aguda, sendo mais comuns as dores de origem gastrointestinal. Os equinos com dor intensa passam a ter atitudes como deitar e levantar constantemente, rolar, e até mesmo de se jogar no chão ou ter dificuldades para se locomover. Apesar da relativa facilidade de se identificar um equino portador de síndrome cólica, torna-se difícil determinar a origem da dor e os fatores que desencadearam este quadro clínico, frente as mais diversas etiologias e a variância de cada caso. A multiplicidade das causas, a complexidade dos casos clínicos e o alto índice de insucesso nos tratamentos, principalmente daqueles que demandam procedimentos cirúrgicos, são apenas algumas das dificuldades na resolução dos casos (LARANJEIRA; ALMEIDA, 2008).

A espécie equina é extremamente exigente e sensível às alterações de manejo alimentar e ambiental. A intensidade das atividades físicas, alterações súbitas na dieta e nas condições de estabulação, a ingestão de dietas ricas em concentrados, os volumosos ou concentrados de má qualidade, e o consumo excessivamente rápido do concentrado, a privação de água e o longo transporte em viagens, podem desencadear a episódios de Cólica (HILLYER et al., 2001).

O quadro de abdômen agudo pode ser ocasionado por diversas enfermidades, envolvendo as diferentes porções do trato gastrintestinal. Os distúrbios podem ser gástricos ou intestinais, obstrutivos ou não, com ou sem estrangulamento vascular. O prognóstico dependerá de vários fatores, como o tempo de evolução, a região afetada e o grau de comprometimento do órgão envolvido (COHEN, 1997).

As lesões ocorridas nas síndromes cólicas são na maioria das vezes anatômicas, categorizadas funcionalmente como sendo, obstrutiva, estrangulante, infarto não estrangulante, enterite, peritonite, ulceração ou ileus (TINKER et al., 1997a). A cólica espasmódica ou gasosa, provavelmente, é a causa mais comum delas (COHEN et al., 1999).

A enterite aguda é uma doença inflamatória do intestino delgado, ocasionando manifestações de dor abdominal, podendo também ser denominada como duodeno-jejunite proximal, enterite proximal, enterite anterior ou gastroduodenojejunite. Ela é denominada como uma inflamação catarral no segmento proximal do intestino delgado, envolvendo principalmente o duodeno ( HUSKAMP, 1985; LEETH; ROBERTSON, 1989 ). As taxas de sobrevivência relatadas na literatura variam de 25 a 94\% ( JOHNSTON; MORRIS, 1987; 
SEAHORN; CORNICK; COHEN, 1992; FERNANDES et al., 2003). A etiologia da enterite proximal ainda é desconhecida, porém, a Salmonella e/ou Clostridium são citados como sendo os principais agentes etiológicos (HENNINGER, 1986; WHITE et al., 1987).

Os animais com enterite, muitas vezes apresentam um histórico de ingestão de grãos ou ração peletizada em sua dieta, sugerindo que a enterite aguda possa ser uma lesão inflamatória não-infecciosa do intestino delgado causada por micotoxinas ou por uma fermentação excessiva ou indevida de carboidratos (COHEN et al., 2005).

A enterite caracteriza-se, clinicamente por sinais que vão de leves a severos de dor abdominal, que diminuem após a descompressão gástrica; apresentam febre baixa; refluxo gástrico abundante ou persistente de coloração castanha-alaranjada, de odor fétido; ligeira distensão do intestino delgado; desidratação, endotoxemia e acidose metabólica (WHITE et al. 1987; JOHNSTON et al., 1987; DAVIS et al., 2003).

As alterações anatomopatológicas observadas nesses casos sugerem que a inflamação ocasione efeitos deletérios na motilidade intestinal e nos mecanismos de transporte de água e eletrólitos, causando hipersecreção, redução da absorção e aumento de permeabilidade vascular, entérica e intraluminal. Isso resulta em acúmulo de líquido com distensão do segmento proximal do trato gastrintestinal e distensão gástrica secundária, refletindo em dor e redução da motilidade. Com a evolução do processo, há o desenvolvimento de quadro de desidratação com azotemia, acidose metabólica, choque hipovolêmico, sendo estes complicados por endotoxinas (FREEMAN, 2000).

Macroscopicamente, o intestino delgado apresenta-se distendido e edemaciado, variando de leve a severo, e a sua serosa pode apresentar hemorragias petequiais difusas e equimoses, ou apenas hiperemia. Os achados histopatológicos incluem edema da submucosa, infiltração neutrofílica da submucosa e lâmina própria. Em casos graves, observa-se edema transmural com ulceração da mucosa, hemorragia extensa acompanhada por deposição de fibrina na serosa e necrose. As lesões frequentemente afetam a metade proximal do intestino delgado, porém, ocasionalmente, toda a extensão do intestino delgado e partes do cólon ascendente são afetados. Raramente só a porção distal do intestino delgado está envolvida (BARTON, 2005).

O tratamento conservativo para os casos de enterite proximal é indicado. Neste inclui a descompressão gástrica através de sondagem nasogástrica, evitando assim, a ruptura e aliviando parcialmente a dor gástrica; a administração intravenosa de fluidos isotônicos balanceados para combater a desidratação, choque hipovolêmico, distúrbios eletrolíticos e a azotemia pré-renal; e o uso sistêmico de antiinflamatório não esteróide e antibiótico, quando 
há manifestação clínica da endotoxemia e/ou sepse. (BYARS; SEMRAD, 1983; JOHNSTON; MORRIS, 1987). Deve-se manter o animal sob jejuns hídrico e alimentar. Os fármacos prócinéticos também são utilizados, no entanto, seus resultados são duvidosos, sendo de difícil avaliação qual foi o principal fator envolvido no restabelecimento do peristaltismo. Entre eles, os mais utilizados são a metoclopramida e a lidocaína, geralmente associadas à administração de cálcio (FREEMAN, 2000).

Embora a enterite proximal seja uma afecção algumas vezes controlada clinicamente nos casos leves e moderados, já nos casos graves, ou quando não se consegue distinguir de um processo estrangulativo (JOHNSTON; MORRIS, 1987), a laparotomia exploratória deve ser considerada (BARTON, 2005).

As principais complicações observadas são a laminite (SCHUMACHER, COHEN; DEAHORN, 1994; FREEMAN, 2000; FERNANDES et al., 2003), e a endotoxemia (FREEMAN, 2000).

Além dos fatores desencadeantes como, o rolamento do animal ao solo, da interrelação anatomotopográfica das vísceras abdominais e do peristaltismo aumentado, os deslocamentos do cólon maior podem ocorrer secundariamente a outras afecções dolorosas do abdômen. Nem sempre refletem em estrangulamentos dos vasos e do intestino acometido, podendo ocorrer o deslocamento dorsal à direita ou à esquerda, a destroflexão, retroflexão ou até mesmo o vólvulo do cólon maior. O tratamento envolve a reposição da volemia, eletrolítica e do equilíbrio ácido-base. Para os casos de retroflexão, o tratamento definitivo é a correção cirúrgica através da laparotomia exploratória (THOMASSIAN, 2005).

\section{RELATO DE CASO}

Uma égua da raça Quarto de Milha de 6 anos de idade, pesando 600kg, mantida em sistema extensivo, amanheceu no haras de origem apresentando sinais de desconforto abdominal, como olhar para o flanco, deitar e rolar. No local, foi administrado flunixim meglumine pela via intramuscular e vermífugo via oral. Realizou-se a sondagem nasogástrica, sendo esta produtiva, obtendo-se um refluxo de aproximadamente 7 litros, pela palpação retal, nada foi identificado e, na paracentese, obteve-se um líquido peritoneal sanguinolento. $\mathrm{O}$ animal então foi encaminhado para o Hospital Veterinário da FAFRAM, para avaliação. Ao exame clínico, a égua apresentou temperatura a 37,2 $2^{\circ}$; frequência cardíaca de 49 bpm; pulso fraco; frequência respiratória de $16 \mathrm{mpm}$; TPC de 3 a 4 egundos, e hipermotilidade no antímero esquerdo e hipomotilidade no antímero direito. Foi observado presença de halo 
toxêmico gengival. Na sondagem nasogástrica, obteve-se um líquido amarelado de aproximadamente 5 litros, apresentando $\mathrm{pH}$ 7,0. Nos exames hematológicos e bioquímicos realizados, foi observado apenas hemoconcentração. Na palpação retal, o intestino delgado encontrava-se palpável e com uma discreta distensão; tensão na raiz mesentérica; exacerbada sensibilidade no ceco com possível deslocamento e a flexura pélvica não foi localizada. A paracentese foi improdutiva. $\mathrm{O}$ animal foi submetido à fluidoterapia para restabelecer o quadro circulatório, sendo encaminhado à laparotomia exploratória.

$\mathrm{O}$ animal foi submetido à laparotomia exploratória, sob indução anestésica com quetamina e diazepam e manutenção com isofluorano, então procedeu-se a celiotomia retroumbilical mediana para acessar a cavidade abdominal. Após inspeção de toda a cavidade, observou-se deslocamento dorsal-medial do ceco, retroflexão do cólon esquerdo com áreas sugestivas de estrangulamento já desfeito próximas à flexura pélvica. No intestino delgado observou-se edema generalizado, com presença de hematomas e petéquias presentes na serosa, em toda extensão do órgão (Figura 1) e distensão do duodeno. O intestino foi reposicionado em sua posição anatômica, realizando sutura da musculatura com fio vicryl $\mathrm{n}^{\circ}$ 2 em padrão de sutura sultan. O subcutâneo foi reduzido com sutura intradérmica com o vicryl $\mathrm{n}^{\circ} 0$ e a dermorrafia em padrão de sutura simples separado com fio de nylon $\mathrm{n}^{\circ} 0,40$.

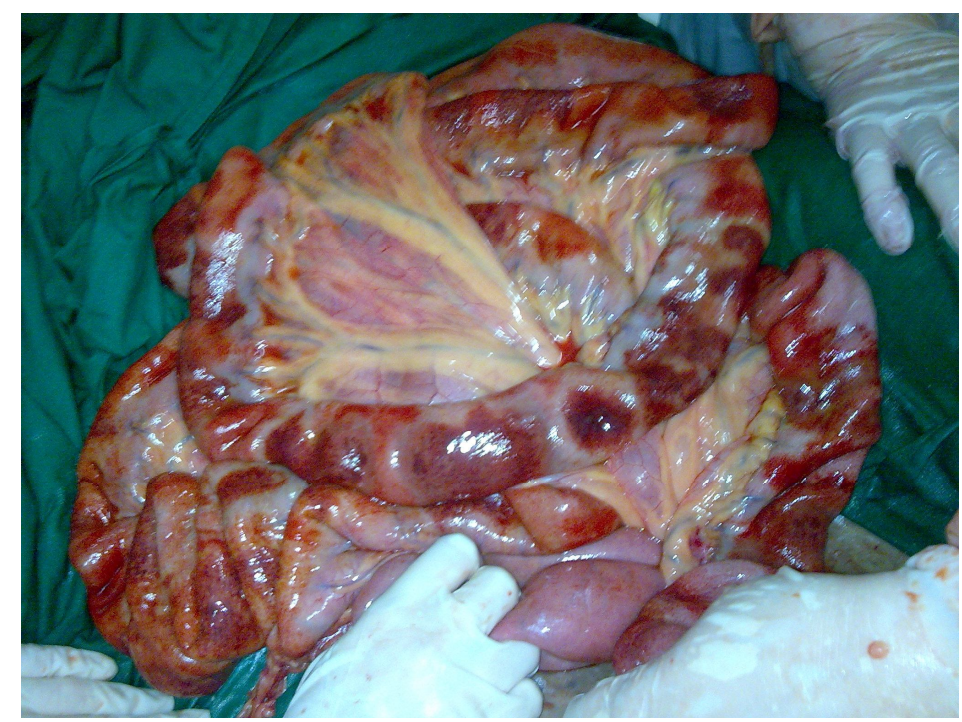

Figura 1 - Presença de hematomas e petéquias na serosa do intestino delgado.

No pós-operatório imediato a égua demonstrou um episódio agudo de desconforto abdominal, sendo administrada a lidocaína $(1,3 \mathrm{mg} / \mathrm{kg})$ intravenosa por infusão contínua até a normalização do quadro de dor e redução da frequência cardíaca. O pós-operatório, foi realizado a base de fluidoterapia intravenosa com Ringer Lactato por 5 dias; Flunixim 
Meglumine $(0,25 \mathrm{mg} / \mathrm{kg})$ TID por 15 dias; DMSO (1g/kg) SID por 5 dias; Penicilina Procaína (44000 UI/kg) BID por 10 dias; Metronizadol (15mg/kg) BID por 3 dias; Gentamicina $(2,2 \mathrm{mg} / \mathrm{kg})$ BID por 3 dias; Meloxicam $(0,6 \mathrm{mg} / \mathrm{kg})$ BID por 8 dias; Cloridrato de Ranitidina (1,5mg/kg) TID por 15 dias); Heparina (40 UI/kg) BID por 20 dias e após, SID por 5 dias; Lidocaína $(1.3 \mathrm{mg} / \mathrm{kg})$ em infusão intravenosa durante os episódios de hipomotilidade e/ou atonia intestinal. Vitamina B12, SID por 3 dias. Com 4 dias de pós-operatório, o animal demonstrou, após ingestão de feno desidratado, sinais de desconforto abdominal, sendo necessária e sondagem nasogástrica e lavagem gástrica. Após este episódio de desconforto gástrico, manteve-se apenas o feno hidratado na dieta do animal; sendo inserido o Omeprazol $(0,7 \mathrm{mg} / \mathrm{kg}) \mathrm{BID}$, junto a terapia medicamentosa.

Foi detectado, 5 dias após a cirurgia, a presença de pulso digital, instituindo a crioterapia podal intensiva nos quatro membros. Nos 10 dias de pós-operatório ainda havia a presença de discreto pulso digital palmar, instituindo-se a Acepromazina $(0,05 \mathrm{mg} / \mathrm{kg}) \mathrm{BID}$ no total de 7 dias, sendo sua frequência reduzida após o quinto dia, concomitantemente ao uso de botinhas de isopor palmar.

\section{RESULTADOS E DISCUSSÃO}

As lesões macroscópicas encontradas em toda a extensão do intestino delgado durante a laparotomia foram atípicas, sendo estas compatíveis com um quadro de enterite, possivelmente enterite proximal, sem etiologia definida, concordando com os achados de Barton 2005, que descreve a enterite proximal como um quadro de edemaciação do intestino delgado e serosa apresentando hemorragias petequiais difusas e equimoses.

Apesar do tratamento da enterite proximal, citado na literatura, ser conservativo em casos leves e moderados que geralmente são responsivos a terapia clínica, optou-se pela laparotomia exploratória em virtude dos achados encontrados na palpação retal, concomitantes à clínica apresentada pelo animal, sugestiva de deslocamento de cólon esquerdo, e também pela possibilidade de uma torção intestinal, evidenciadas pelo quadro circulatório, baixa temperatura e paracentese sanguinolenta.

De acordo com Thomassian (2005), quando alças intestinais pouco repletas em sua capacidade total de contenção de digesta ou com sobrecarga de alimentos com baixo teor de fibras e conteúdo de pouca consistência, poderá ocorrer perda da relação anatomotopográfica por aumento dos movimentos peristálticos, ou decorrentes do próprio rolamento do animal no solo. A retroflexão é detectada à palpação retal pelo posicionamento sagital no sentido crânio- 
caudal da porção medial do cólon dorsal e ventral esquerdo em direção à flexura pélvica. É caracterizada por uma dobra do cólon maior esquerdo em um ângulo de aproximandamente 180 graus no sentido crânio-caudal, sendo este apenas palpável apenas em casos de distensões intestinais.

Optou-se pela utilização da lidocaína sem epinefrina no pós-operatório pois, além de possuir propriedades pró-cinéticas a nível de duodeno, ela também possui propriedades analgésicas, inibindo os efeitos negativos da dor sobre a motilidade intestinal. $\mathrm{O}$ seu efeito analgésico é alcançado pelo bloqueio dos canais de sódio, inibindo os reflexos simpáticos e permitindo o peristaltismo espontâneo. Em doses menores, a lidocaína pode modular a resposta inflamatória, a qual contribui para a redução da contração da músculatura lisa e consequentemente a redução da motilidade. Os anestésicos locais podem melhorar a motilidade, reduzindo a ação, adesão e migrações de neutrófilos (COOK, 2009).

A complicação pós-operatório de destaque observada foi a endotoxemia, concordando com Freeman (2000), que cita este quadro como uma das complicações mais comuns da enterite proximal. A terapia instituída, incluindo flunixim meglumine $(0,25 \mathrm{mg} / \mathrm{kg}) \mathrm{TID}$, foi eficiente para controlar a endotoxemia, associada a antibioticoterapia sistêmica e fluidoterapia.

O desconforto abdominal apresentado após 4 dias do procedimento cirúrgico reflete um quadro de gastrite e irritação da mucosa gástrica, ocorrido após ingestão de feno, sendo este retirado da alimentação do animal, fornecendo apenas feno hidratado.

A presença de pulso digital também verificada no pós-operatório sugere um início de laminite aguda, concordando com Schumacher et al. (1994), Freeman (2000) e Fernandes et al. (2003) que afirmaram a laminite como a principal complicação da enterite proximal. O uso da crioterapia podal para controle da laminite, concorda com Pollitt et al (2003), que cita este método como eficaz no tratamento desta afecção. O profundo efeito hipometabólico causado pela crioterapia pode ser o mecanismo mais importante para limitar a gravidade das lesões, pois a taxa metabólica e de consumo de oxigênio são inversamente proporcionais à temperatura. A menor necessidade em oxigênio, glicose e outros metabólicos dos tecidos sujeitos a crioterapia diminuem as lesões celulares resultantes de períodos de isquemia. A atividade das colagenases e citocinas pró-inflamatórias também é diminuída a temperaturas baixas (POLLITT, 2007).

Após o término da terapia medicamentosa e retirada dos pontos cirúrgicos, os sinais de desconforto abdominal, endotoxemia e laminite cessaram, refletindo na alta hospitalar do animal. 


\section{CONCLUSÃO}

A terapia clínica e cirúrgica foi satisfatória, apesar das complicações pósoperatórias apresentadas pelo animal.

Nem sempre é possível tratar clinicamente uma enterite anterior, quando esta ocorre concomitantemente a outra afecção intestinal, justificando a realização da laparotomia exploratória para correção cirúrgica da enfermidade.

\section{REFERÊNCIAS}

BARTON, M.H. Diagnosis and treatment of enteritis and inflammatory bowel disease. College of Veterinary Medicine, The University of Georgia, Athens, Georgia, 2005.

BYARS, T.D., SEMRAD, S.D. Management of some types of colic. Modern Veterinary Practice, p. 917-921, 1983.

COHEN, N. D. Epidemiology of colic. Veterinary Clinics of North America: Equine Practice, v. 13, n. 2, p. 191-201, 1997.

COHEN, N. D.; GIBBS, P. G.; WOODS, A. M. Dietary and other management factors associated with colic in horses. Journal of American Veterinary Medical Association, v. 215, n. 1, p. 53-60, 1999.

COHEN, N.D.et al. Is duodenitis=proximal jejunitis associated with high carbohydrate diets? Proceedings from the Eight International Equine Colic Research Symposium. Quebec City, Canada, Aug. 3-5, 2005.

COOK, V.L; Gastrointestinal prokinetic drugs. In: ROBINSON, N. E.; SPRAYBERRY.

Current therapy in equine medicine. 6. ed. St. Louis, USA: Saunders, 2009, p.389- 391.

DAVIS, J.L.et al. A retrospective analysis of hepatic injury in horses with proximal enteritis (1984-2002). 2003.

FERNANDES, W. R.et al. Revisão de 26 casos clínicos de duodeno-jejunite proximal em equinos (1996-2000). Ciência Rural, v. 33, n. 1, p. 97-102, 2003.

FREEMAN, D.E. Duodenitis-proximal jejunitis. Equine Veterinary Education, v.12, n.6, p.322-332, 2000.

HENNINGER, R.W. Proximal enteritis in a quarter horse stallion. Compendium of Continuing Education for the Practing Veterinarian, v.8, n.2, p.S53-S58, 1986.

HILLYER, M. H.; TAYLOR, F. G. R.; FRENCH, N. P. A cross-sectional study of colic in horses on Thoroughbred training premises in the British Isles in 1997. Equine Veterinary Journal, v. 33, n. 4, p. 380-385, 2001. 
HUSKAMP, B. Diagnosis of gastroduodenojejunitis and its surgical treatment by a temporary duodenocaecostomy. Equine Veterinary Journal, v.17, n.4, p.314-316, 1985.

JOHNSTON, J.K.; MORRIS, D.D. Comparison of duodenitis/proximal jejunitis and small intestinal obstruction in horse: 68 cases (1977-1985). Journal of American Veterinary Medical Association, v.191, n.7, p.849-854, 1987.

LARANJEIRA, P.V.E.H; ALMEIDA, F.Q. Síndrome cólica em equinos: ocorrência e fatores de risco. Rev. de Ciên. da Vida, RJ, EDUR. v. 28, n. 1, p. 64-78, 2008.

LEETH, B.; ROBERTSON, J. A retrospective comparison of surgical to medical management of proximal enteritis in the horse. In: ANNUAL CONVENTION OF THE AMERICAN ASSOCIATION OF EQUINE PRACTITIONERS, 34, 1988. Proceedings..., 1989, p.69-79.

POLLITT, C.C.et al. Equine Laminitis [versão eletrônica]. 2003. Proceedings... New Orleans, USA, 21-25 November. Disponível em:

<http://www.ivis.org/proceedings/AAEP/2003/pollitt/IVIS.pdf> Acesso em: 11 nov. 2007.

POLLITT, C.C. (2007). Update on the pathophysiology of laminitis [versão electrônica]. In: CHUIT, P.; MONTAVON, S. Proceedings... In: GENEVA CONGRESS OF EQUINE MEDICINE AND SURGERY, 10. Geneva, Switzerland, 11-13 December, p.12-15.

Disponível em : <http://www.ivis.org/proceedings/geneva/2007/p012_017_Pollitt.pdf> Acesso em: 20 fev. 2010.

REED, S.M; BAYLY, W.M. Medicina interna equina, Rio de Janeiro: Guanabara Koogan, 2000, p. 565.

SCHUMACHER, J.; COHEN, N.D.; DEAHORN, T.L. Duodenitis/Proximal Jejunitis in Horses. Compendium of Continuing Education for the Practing Veterinarian, v.16, n.9, p.1197-1206, 1994.

SEAHORN, T.L.; CORNICK, J.L.; COHEN, N.D. Prognostic indicators for horses with duodenitis-proximal jejunitis. Journal of Veterinary Internal Medicine, v.6, n.6, p.307-311, 1992.

TINKER, M. K.; WHITE, N. A.; LESSARD, P.; THATCHER, C. D.; PELZER, K. D.; DAVIS, B.; CARMEL, D. K. Prospective study of equine colic incidence and mortality. Equine Veterinary Journal, v. 29, n. 6, p. 448-453, 1997a.

WHITE, N.A.; TYLER, D.E.; BLACKWELL, R.B.; et al. Hemorrhagic fibrinonecrotic duodenitis-proximal jejunitis in horses: 20 cases (1977-1984). Journal of American Veterinary Medical Association, v.190, n.3, p.311-315, 1987.

WHITE II, N.A. Equine Colic. AAEP Proceedings, v.52, 2006, p.109-174. 
